Cahiers de civilisation médiévale

\title{
Collectif, Medieval and Early Modern Murder: Legal, Literacy and Historical Contexts
}

\section{Valérie Toureille}

\section{OpenEdition}

1 Journals

Édition électronique

URL : https://journals.openedition.org/ccm/5403

DOI : $10.4000 / \mathrm{ccm} .5403$

ISSN : 2119-1026

Éditeur

Centre d'études supérieures de civilisation médiévale/Université de Poitiers

\section{Édition imprimée}

Date de publication : 1 décembre 2020

Pagination : 291-293

ISBN : 978-2-490783-07-6

ISSN : 0007-9731

\section{Référence électronique}

Valérie Toureille, "Collectif, Medieval and Early Modern Murder: Legal, Literacy and Historical Contexts ", Cahiers de civilisation médiévale [En ligne], 252 | 2020, mis en ligne le 02 décembre 2020, consulté le 09 décembre 2022. URL : http://journals.openedition.org/ccm/5403; DOI : https://doi.org/10.4000/ccm. 5403

\section{cc)}

Creative Commons - Attribution - Pas d'Utilisation Commerciale - Pas de Modification 4.0 International - CC BY-NC-ND 4.0

https://creativecommons.org/licenses/by-nc-nd/4.0/ 
Medieval and Early Modern Murder: Legal, Literacy and Historical Contexts, L TRACY (dir.), Woodbridge, Boydell Press, 2018.

Cet ouvrage réunit vingt contributions sous la direction de Larissa Tracy, professeur de littérature médiévale à l'université de Longwood (EU). Son ambition est de brosser le panorama général d'un crime en particulier : le meurtre depuis les premiers siècles du Moyen Ầge jusqu'au XVI ${ }^{\mathrm{e}} \mathrm{s}$. C'est une synthèse intéressante, difficile et ambitieuse. Après avoir introduit le propos, L. Tracy articule sa démonstration autour de trois parties. La première explore le cadre juridique propre à examiner des affaires de meurtres dans différents types de sources. La deuxième examine «l'herméneutique publique» du meurtre. Autrement dit, comment les sociétés médiévales appréhendent-elles le meurtre à travers leur production littéraire, partant des sagas islandaises jusqu'aux fabliaux français en passant par la tradition arthurienne. La troisième et dernière partie porte sur les effets du meurtre sur la communauté, perçu comme une «maladie sociale», en particulier pour les meurtres intrafamiliaux. Ce découpage thématique introduit une progression chronologique au sein de chaque partie.

L. Tracy débute son introduction en présentant le meurtre comme la grande angoisse des sociétés humaines depuis des siècles, et de citer à l'appui le plus ancien recueil de lois connu : le code sumérien d'Ur-Nammu. Certes, la première disposition condamne le meurtrier à mort, mais c'est oublier que le code met sur le même pied le voleur qui doit aussi être condamné à la peine capitale. Par ailleurs, l'autre grand recueil de lois, le code babylonien de Hammourabi, un peu plus récent, ne contient aucune disposition sur le meurtre, à la différence du vol qui est là encore châtié par la mort. L'a. souligne en revanche avec pertinence que le meurtre permet de lire "en creux» les liens qui unissent ou désunissent la famille et plus encore, toutes les relations sociales. Dans la société contemporaine, le meurtre fascine et inquiète. Il nourrit la littérature et plus abondamment encore le cinéma. Mais cette angoisse était-elle partagée par les hommes et les femmes du Moyen Âge? On peut répondre, à l'instar de l'a., par la négative. Les homicides (par définition involontaires) sont nombreux, mais les meurtres ou assassinats crapuleux sont rares. L. Tracy revient à ce titre sur la définition de ces termes : exigeant la préméditation, donc la volonté délibérée de tuer, parfois accompagnée de circonstances aggravantes. L'a. entend se situer dans le sillage des travaux de Trévor Dean, mais en centrant sa démarche sur le meurtre de façon exclusive. Les différents chapitres de ce livre entendent revenir sur la définition du meurtre, de l'homicide volontaire ou non, justifié ou pas, des considérants qui dépendent étroitement de la contextualisation des dispositions juridiques, mais l'on pourrait aussi ajouter par la manière dont la transgression est reçue par la communauté à un moment donné. L'a. pousse son argumentaire plus 
loin (peut-être trop) en affirmant que les lois (celles qui ont, en particulier, réprimé le meurtre) et le spectacle des exécutions ont agi conjointement comme un catalyseur de la conscience nationale : «Laws acts as a catalyst or dynamic to a consciousness of "nationality"» (p. 7).

L. Tracy résume ainsi son approche: au Moyen Âge, le meurtre est appréhendé à travers différents paramètres qui ont évolué avec le temps, la culture, l'espace et la loi. Ce volume entend donc saisir la grande variété des circonstances qui entourent le crime pour mieux le définir, c'est-à-dire à travers les textes de loi, la littérature, les châtiments et les prohibitions. Mais également à travers les réponses sociale et jurisprudentielle qui lui sont apportées. L'a. place ainsi sous son microscope tous les types de meurtres : assassinat, infanticide, maricide (sic), régicide, tyrannicide comme le simple homicide. Mais n'y a-t-il pas d'emblée un problème de définition, que l'on retrouve au fil des pages? Pourquoi ne pas privilégier l'approche des jurisconsultes médiévaux qui se sont naturellement penchés sur la question de la définition du meurtre et de l'homicide, en soulignant une différence notable introduite par la prise en compte de l'intention, ce que notre droit reconnaît toujours, par ailleurs.

La première partie («Murder on Trial: Justice, Law and Society») appréhende le meurtre à travers une grande variété de sources incluant des procès, des textes juridiques et littéraires pour en définir le cadre légal. Bridgette Slavin (Univ. Sydney) présente ainsi des récits de meurtres et de magie, à la fois dans la littérature irlandaise ancienne et dans les textes normatifs. Jay Paul Gates (Univ. Wisconsin) s'efforce d'exposer les implications sociopolitiques des meurtres de masse dans le discours littéraire du Beowulf, et l'exemple du massacre de la saint Brice par le roi anglo-saxon Æthelred (1002). Pinchas Roth (Univ. Jérusalem) étudie les lois talmudiques du Moyen Âge (Halakhah) et les dispositions préconisées pour la condamnation et l'exécution des meurtriers. Jolanta N. Komornicka (Univ. Boston) analyse le vocabulaire utilisé dans les procès qui se sont tenus devant le Parlement de Paris entre 1312 et 1320 mettant aux prises les meurtriers avec l'incrimination de trahison. L. Tracy privilégie, quant à elle, les contes de Canterbury de Chaucer pour cerner l'exercice de la justice. L'on y trouve de nombreux récits se rapportant à des meurtriers condamnés grâce au travail des instances judiciaires ou par l'intervention divine qui perce à jour leur secret.

La deuxième partie («The Public Hermeneutics of Murder: Interpretation and Context») mêle des études qui s'appuient exclusivement sur la littérature et d'autres sur des actes de la pratique judiciaire. Les Sagas islandaises sont ici mises en valeur par Isle Schweitzer Van Donkelaar (Univ. W. Michigan) pour retrouver à travers une série de récits fantastiques des figures de meurtriers et des exemples d'exécutions. Anne Latowski (Univ. Washington) a, pour sa part, choisi de partir des fabliaux français pour reconstituer un discours sur le meurtre, par l'intercession de la comédie. Dans le cycle arthurien, et en particulier, dans La Queste du Saint Graal, Lucas Wood (Univ. Pennsylvanie) s'efforce de déchiffrer la morale de la chevalerie face à sa brutalité et sa violence inhérentes. Dwayne C. Coleman (Univ. Arkansas) prolonge cette réflexion autour de la Morte d'Arthur. Le deuxième volet adopte une posture un peu différente, à l'image de l'article de Matthew Lubin (Univ. N. Carolina) qui propose d'étudier l'empoisonnement comme un type d'exécution politique dans la République de Venise entre la fin du Moyen Âge et le monde moderne à partir de la jurisprudence. Ou l'approche d'Emily Hutchinson (Univ. de York) qui reprend un dossier judiciaire remarquable et singulier, celui de l'assassinat du duc d'Orléans en 1407. Celui-ci perdit non seulement la vie, mais aussi son honneur pour avoir été vilipendé comme tyran par son assassin le duc de Bourgogne, qui justifia ainsi le recours à la violence. Andrew McKenzie-Mc Harg (Univ. Erfurt) examine toutes les stratégies pour diffamer les Jésuites à travers la théorie du complot, et leur complicité dans une série d'assassinats.

La troisième partie aborde la question du meurtre par le prisme du Gender, mais également de l'âge. Elle explore aussi le cœur de la famille et ses fractures dont le meurtre peut-être le révélateur. Deux études sont consacrées à la période du haut Moyen Âge, l'une à travers une source narrative, l'autre par le truchement d'une source normative : Jeffrey Doolittle (Univ. Fordham) examine ainsi les arbitrages autour des cas de meurtres dans l'Histoire des Francs de Grégoire de Tours. Thomas Gobbitt (Univ. Leeds) analyse les dispositions juridiques en cas d'empoisonnement et de meurtre contenues dans l'Édit de Rothari. G. Koolemans Beynen (Univ. Stanford) nous fait entrer dans l'univers inattendu d'un poème caucasien du XIII ${ }^{\mathrm{e}} \mathrm{s}$. rédigé en géorgien ancien (In the Leopard Skin), où il est aussi question de meurtre. Carmel Ferragud (Univ. Valencia) analyse divers cas d'empoisonnement dans la cité de Valence en 1442 à travers le récit qu'en donnent les complaintes littéraires, qui portent l'essentiel de cette mémoire judiciaire. Patricia Turning (Univ. California) étudie de manière efficace le parcours de Claire de Portet 
accusée du meurtre de son mari (un cas de "maricide" dans la cité de Toulouse au début du XV $\mathrm{Xv}^{\mathrm{e}} \mathrm{s}$.), à un moment où d'autres cas sont criminalisés pour l'exemple. Les deux derniers articles, un peu en décalage, s'inscrivent dans une chronologie plus récente avec l'évocation d'infanticides dans la littérature anglaise moderne (Diane Berg, Univ. Tufts) et le rôle des jeunes dans les meurtres rapportés par la première presse anglaise à la fin du XVII ${ }^{\mathrm{e}} \mathrm{s}$. (Ben Parsons, Univ. Sheffield). L'ouvrage est enfin rassemblé dans une synthèse sous la plume d'Hannah Skoda (Univ. Oxford). Elle réussit le pari audacieux de reprendre les interrogations initiales, en revenant sur les questions de définition, tout en mettant l'accent sur le secret et son incidence sur la qualification du geste criminel mortel.

Au-delà de ses approches variées et fort intéressantes que donne à lire cet épais recueil sur le meurtre au
Moyen Âge, on peut simplement émettre quelques regrets, comme il en existe toujours dans les ouvrages synthétiques. On aurait aimé voir figurer des éditions de sources scientifiquement reconnues parfois oubliées par certains auteurs. Les sources littéraires d'ailleurs sont souvent privilégiées sur les actes de la pratique ou les sources normatives, mais il est vrai que la période du haut Moyen Âge n'offre pas de sources de juridiques nombreuses qui permettraient de rétablir la balance. La bibliographie française est quelques fois lacunaire, ce qui est dommageable pour certaines études portant sur des sources médiévales françaises.

Valérie ToureILlE CY Cergy-Paris Université 